\title{
Mise en service d'une nouvelle autoroute et modification des flux de circulation : le cas de l'A
}

39

Opening of a new highway and change of traffic flows : the A 39 case

Inbetriebnahme einer neuen Autobahn und Veränderung der Verkehrsflüsse :

das Beispiel der A 39

Daniel Mathieu

\section{OpenEdition}

Journals

Édition électronique

URL : http://journals.openedition.org/rge/4276

DOI : $10.4000 /$ rge.4276

ISSN : 2108-6478

Éditeur

Association des géographes de l'Est

Édition imprimée

Date de publication : 1 septembre 1999

ISSN : 0035-3213

Référence électronique

Daniel Mathieu, «Mise en service d'une nouvelle autoroute et modification des flux de circulation : le cas de l'A 39 », Revue Géographique de l'Est [En ligne], vol. 39 / 4 | 1999, mis en ligne le 12 août 2013, consulté le 08 septembre 2020. URL : http://journals.openedition.org/rge/4276 ; DOI : https://doi.org/ $10.4000 /$ rge. 4276

Ce document a été généré automatiquement le 8 septembre 2020.

Tous droits réservés 


\title{
Mise en service d'une nouvelle autoroute et modification des flux de circulation : le cas de l'A 39
}

\author{
Opening of a new highway and change of traffic flows : the A 39 case \\ Inbetriebnahme einer neuen Autobahn und Veränderung der Verkehrsflüsse : \\ das Beispiel der A 39
}

Daniel Mathieu

1 Le 2 juin 1998 était inaugurée la mise en circulation des $110 \mathrm{~km}$ de l'autoroute A 39 reliant Dole à Bourg-en-Bresse, via Poligny et Lons-le-Saunier. Cette ouverture complète la mise en service, en novembre 1994, d'un premier tronçon entre Dijon et Dole. Ainsi est créé un nouvel axe de circulation à l'est des plaines de la Saône, dont la vocation est de servir d'itinéraire alternatif à l'autoroute A 6, menacée de saturation entre Beaune et Lyon. Outre sa fonction de délestage, l'A 39 devrait absorber une partie $\mathrm{du}$ trafic utilisant les voies routières méridiennes existantes, compte tenu des gains de temps et de sécurité qu'elle procure par rapport au réseau traditionnel. Enfin, l'amélioration des conditions locales de l'accessibilité pourrait susciter de nouvelles dynamiques économiques, elles-mêmes génératrices de trafic.

La Société des Autoroutes Paris-Rhin-Rhône (SAPRR), à qui a été confiée la concession des travaux et de l'exploitation, a créé, en partenariat avec le ministère de l'Équipement et les trois conseils généraux des départements concernés par le tracé (Ain, Jura, Saône-et-Loire), un Observatoire des effets écologiques et socio-économiques de l'autoroute A 39 sur les régions traversées. L'IRADES, laboratoire de l'université de Franche-Comté, s'est vu confier l'ensemble des recherches concernant le volet socioéconomique. Les travaux de l'Observatoire ont commencé en 1993, avant l'ouverture du chantier, et devraient se poursuivre jusqu'en 2002-2003. Parmi les nombreuses thématiques de recherches retenues, une des préoccupations essentielles concerne les modifications de l'organisation des flux de circulation que suscitera la nouvelle 
infrastructure, et les conséquences que ces transformations pourraient avoir sur l'organisation socio-économique des espaces proches de la nouvelle infrastructure.

\section{Méthodologie}

3 L'évaluation des transformations induites par l'ouverture de l'A 39 suppose de pouvoir comparer des états des flux avant et après sa mise en service. Dans cette perspective, le CETE de Lyon a été chargé, pour le compte de la SAPRR, de mettre en œuvre plusieurs enquêtes de circulation afin de répondre aux obligations de la Loi d'Orientation des Transports Intérieurs (LOTI) d'une part et aux besoins de l'Observatoire d'autre part. Près de 16000 interviews ont été réalisées en 1997 par une équipe d'étudiants de l'IRADES. Deux enquêtes ont eu lieu au cours de périodes de départ en vacances, en hiver et en été, pour cerner les flux touristiques. Une troisième, dont nous présentons ici quelques résultats, a été effectuée en mai, un jour de semaine censé représenter les trafics de périodes ordinaires.

4 Pour cette enquête, huit postes ont été définis par le CETE de Lyon : Viriat RN 83, Viriat A 40, Marboz, Villefranche A 6, Tournus A 6, Lons-nord RN 83, Lons-sud RN 83, Lonsouest RN 78. Parmi ces huit sites, les trois derniers présentent un intérêt tout particulier dans la mesure où ils permettent l'analyse des flux aux entrées de Lons-leSaunier. En effet ce secteur, traversé par l'axe nord/sud de la RN 83, connaîtra vraisemblablement d'importantes modifications de l'organisation et de l'intensité des trafics routiers après la mise en service de l'autoroute et du diffuseur de LonsBeaurepaire, situé quelques kilomètres à l'ouest de l'agglomération. Deux postes d'enquête se situent le long de la RN 83, de part et d'autre de Lons-le-Saunier; le sens des flux étudiés est dirigé vers le sud pour le poste de Lons-nord et vers le nord pour le poste de Lons-sud. Enfin, le troisième poste, installé le long de la RN 78 à l'ouest de la ville, recense les trafics en provenance de la Bresse louhannaise et de la vallée de la Saône.

5 Les enquêtes ${ }^{1}$ se sont déroulées entre 7 heures et 19 heures. Compte tenu de l'importance des trafics et du nombre limité d'enquêteurs, il n'a pas été possible d'intercepter tous les véhicules. Le rendement horaire par poste est de l'ordre de 100-150 interviews : le taux de sondage varie donc au cours de la journée en fonction de l'intensité de la circulation. Aussi un recensement manuel effectué parallèlement, permet de définir un taux de sondage horaire et ainsi d'étendre les résultats obtenus pour l'échantillon enquêté à l'ensemble des véhicules. Par ailleurs, en utilisant les données de comptages automatiques réalisés pendant les jours ouvrables de la semaine d'enquête, une démarche analogue permet de réajuster les résultats de la période d'enquête, d'une part sur la journée entière, d'autre part sur le trafic journalier moyen constaté durant la semaine dans les deux sens. Mais cette extrapolation de résultats obtenus entre 7 et 19 heures, à un trafic nocturne qui, de toute évidence, ne présente pas la même structure, affecte la fiabilité des données redressées, bien que son poids soit modeste, en général moins de $20 \%$ du total. C'est pourquoi ces données extrapolées à la journée moyenne de la semaine d'enquête ne seront pas utilisées pour la suite de l'analyse, sauf pour établir les bilans globaux et des comparaisons avec les valeurs journalières moyennes établies pour l'ensemble de l'année 1997.

6 Les questionnaires utilisés pour cette opération apportent une très grande richesse d'informations et permettent d'aborder l'état des lieux avant ouverture de l'autoroute 
sous deux aspects. L'un concerne l'organisation des flux de circulation et les potentiels de transfert qu'ils recèlent. L'autre porte sur le système de relations que l'agglomération de Lons-le-Saunier entretient avec les différentes composantes de l'espace régional. Seul le premier point sera développé ici.

\section{La structure générale des flux de circulation}

7 Dans le secteur où la RN 83 est longée par l'autoroute A 39, le trafic est connu grâce aux comptages réalisés régulièrement par les services de l'État. Pour l'année 1997, les moyennes journalières comptabilisant les trafics dans les deux sens varient suivant la localisation des postes de comptage. Ceux-ci intègrent en effet à la fois le transit à moyenne et longue distance, et le trafic local qui est d'autant plus fort que le poste est proche d'une agglomération. Ainsi, à proximité de Lons-le-Saunier, les valeurs sont de 14000 véhicules/jours au nord (dont 2100 poids-lourds, soit $15,6 \%$ du total) et de 17 $800 \mathrm{v} / \mathrm{j}$. au sud. Par comparaison, à la sortie nord de l'agglomération de Bourg-enBresse, le trafic moyen est de $10800 \mathrm{v} / \mathrm{j}$. Enfin, entre les deux agglomérations, là où le trafic local est moins intense, les chiffres les plus faibles sont de $8700 \mathrm{v} / \mathrm{j}$. à Coligny, dont 1900 poids-lourds ( $20,5 \%$ du total). Pour la RN 78, le poste de Lons-le-Saunier ouest donne une moyenne sensiblement inférieure aux plus faibles valeurs de la RN 83 $(6650 \mathrm{v} / \mathrm{j})$; le trafic poids-lourds est également moins bien représenté avec seulement 600 véhicules, soit $9 \%$ du trafic moyen journalier total. Les résultats obtenus lors de la période d'enquête et redressés à la journée de 24 heures, sont quelque peu différents des moyennes annuelles. Ainsi pour les postes de Lons-nord et de Lons-ouest, les chiffres sont légèrement supérieurs à la moyenne, mais la situation est inverse pour Lons-sud, sans doute en fonction de la localisation du poste d'enquête, plus proche de la ville. Enfin pour les trois postes, il faut noter que les pourcentages de poids-lourds sont logiquement plus forts lors de l'enquête que pour la journée moyenne. En effet celle-ci intègre les trafics des week-ends et des périodes de vacances pendant lesquelles les véhicules légers sont très sur-représentés.

Par rapport à la question d'éventuels transferts de trafic de la RN 83 vers l'A 39, l'intérêt des enquêtes est de pouvoir travailler sur une organisation fine des flux en fonction des origines et des destinations des déplacements. Il est clair en effet que l'utilisation de l'autoroute dépendra de la longueur des trajets à parcourir et de leurs directions géographiques. Mais, afin d'obtenir une bonne lisibilité, les originesdestinations très précises fournies par les enquêtés ont été regroupées en trois grands types d'ensembles spatiaux, eux-mêmes subdivisés en deux sous-groupes (fig. 1a et 1b). L'espace local se définit par les cantons proches de l'autoroute, de la région de Dole jusqu'à Bourg-en-Bresse, ainsi que par les marges de la Bresse et de la montagne jurassienne. Pour chaque poste d'enquête, il sera nécessaire de distinguer un espace local nord et un espace local sud dont les configurations géographiques ne seront pas rigoureusement identiques dans les deux cas. L'espace régional, plus éloigné de la région lédonienne, se divise également en deux sous-espaces : l'espace régional-nord correspond grossièrement aux régions dijonnaise et bisontine, l'espace régional-sud couvre l'essentiel de la région Rhône-Alpes. Enfin, l'espace lointain se divise également en deux : l'espace lointain-nord rassemblant les régions de la France du nord, de l'ouest et de l'est ainsi que les pays étrangers de l'Europe du nord-ouest, l'espace lointain-sud associant les régions du sud de la France et les pays de l'Europe méditerranéenne. 
Figure 1a : L'A 39 dans le réseau routier et autoroutier régional

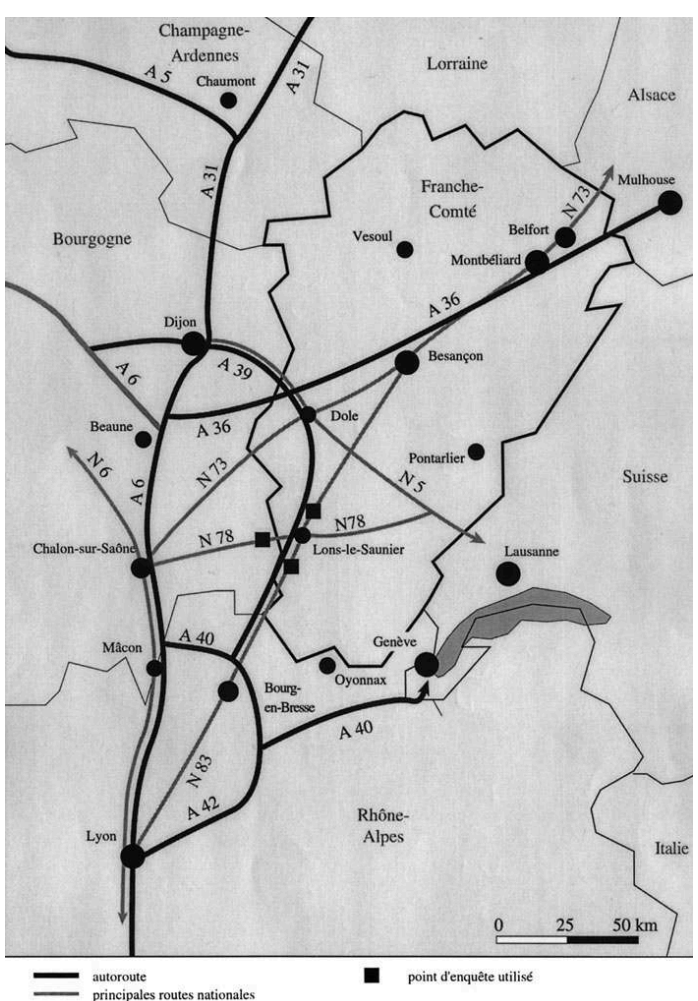

Figure 1b : Le découpage spatial utilisé

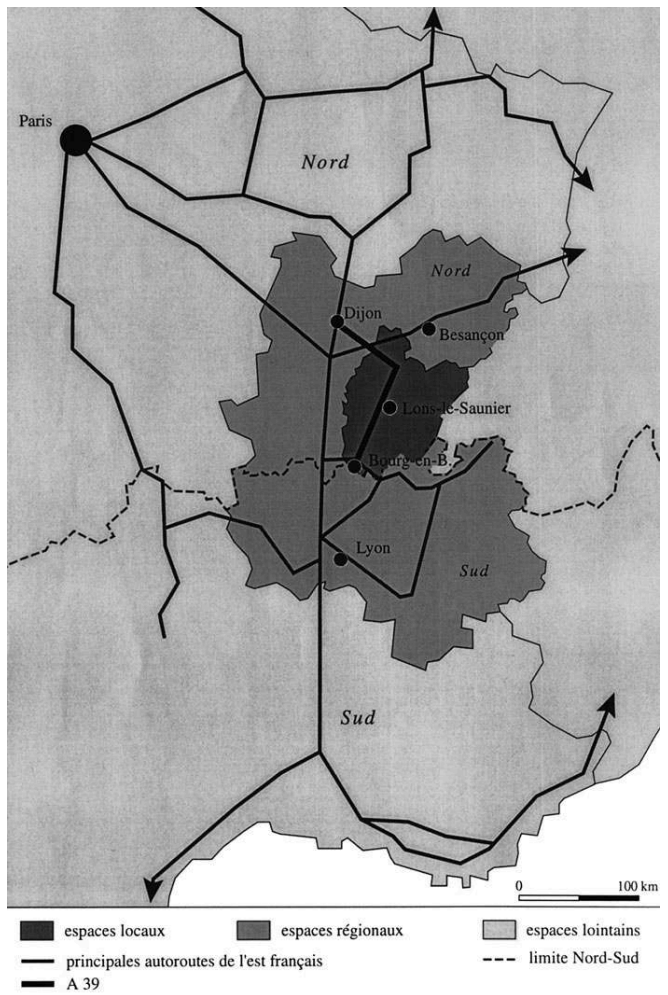

Parmi les trois postes d'enquêtes situés en périphérie de l'agglomération lédonienne, les deux qui sont installés sur la RN 83 sont essentiels car ils concernent des flux nord- 
sud qui intéressent directement l'A 39. Ils feront donc l'objet d'une présentation détaillée de la méthode d'analyse et des résultats. En revanche, seuls les bilans globaux seront fournis pour le poste de Lons-ouest. Enfin, dans la mesure où ils ne répondent pas aux mêmes logiques, les trafics véhicules légers et les trafics poids-lourds seront traités séparément. La méthode de travail étant identique dans les deux cas, elle ne sera développée que pour les véhicules légers.

\section{Les flux de véhicules légers sur la RN 83} les valeurs étant de 4537 véhicules pour Lons-nord et 2880 pour celui de Lons-sud. La non-symétrie des chiffres tient pour une large part aux flux qui ont une destination locale, car la plupart des véhicules s'arrêtent dans l'agglomération et quelques-uns quittent la RN 83 pour se diriger, soit vers la Bresse, soit vers le massif jurassien, par la RN 78. Par ailleurs, si, en théorie, les flux de transit «montants " et «descendants" devraient être à peu près identiques, dans la réalité des écarts plus ou moins importants sont constatés; les différences s'expliquent par le caractère aléatoire de résultats établis sur une seule journée d'observation.

11 L'analyse des données fournies par les enquêtes sera conduite en fonction des types d'espaces que les flux mettent en relation, pour des raisons techniques (les chiffres ne recouvrant pas toujours la même réalité) mais surtout pour pouvoir apprécier, au cas par cas, les possibilités de transfert vers l'A 39.

\section{A. Entre espaces locaux}

ux correspondent, à plus de $90 \%$ pour les deux postes, à des échanges entre Lonsle-Saunier et les communes proches. Pour le poste de Lons-nord, ils représentent 2715 véhicules, soit $61 \%$ du total des VL. Ils se font avec les espaces périurbains et ruraux d'une part, et avec le réseau des villes nord-jurassiennes d'autre part (Dole surtout, et secondairement Poligny, Arbois et Salins). Dans cet espace, seuls les déplacements entre Dole et Lons-le-Saunier pourraient, en théorie, emprunter l'autoroute; dans la réalité cette hypothèse est peu vraisemblable, compte tenu, d'une part, de la position très excentrée de l'échangeur de Beaurepaire par rapport à Lons-le-Saunier, d'autre part, de la bonne desserte assurée entre ces deux villes par la RD 475. Pour le poste de Lons-sud, les flux locaux sont plus limités que précédemment. Ils s'élèvent à 1907 véhicules ( $65 \%$ du total des VL) et relient, dans leur écrasante majorité, Lons-leSaunier et les petites cités franc-comtoises du Revermont. Par ailleurs les relations sont très faibles vers le sud avec le secteur de Bourg-en-Bresse, à la différence de ce qui se passe au nord entre Dole et la capitale jurassienne. Dans tous les cas, l'autoroute ne présente que très peu d'intérêt, compte-tenu des faibles distances à parcourir et de l'aménagement de longues sections à deux fois deux voies sur la RN 83.

\section{B. Entre espaces locaux et espaces régionaux}

Établis sur de plus longues distances que les précédents, ces flux sont beaucoup moins importants et, là encore, très déséquilibrés entre les deux postes d'observation. 
$14 \mathrm{Au}$ poste de Lons-nord, ils représentent environ 900 véhicules. Les relations entre l'espace local-nord (secteurs de Dole, Arbois-Poligny) et la région Rhône-Alpes devraient logiquement emprunter l'A 39, mais ils sont anecdotiques : une cinquantaine de véhicules seulement. L'essentiel du flux s'organise entre Lons-le-Saunier et la capitale franc-comtoise (environ 600 véhicules) ou la capitale bourguignonne (300 véhicules). Le recours à l'A 39, probable dans le second cas, l'est sans doute assez peu dans le premier : à temps de parcours égal, mais sans péage, la liaison avec la région bisontine, par la RN 83, restera sans doute privilégiée, au moins dans un premier temps, d'autant que d'importants travaux d'aménagement sont en cours dans le cadre du XIIe Plan État-Région, en particulier la réalisation d'une nouvelle section à deux fois deux voies pour le contournement de Quingey.

15 Les flux entre espaces régionaux et espaces locaux sont plus ténus au poste de Lons-sud (360 véhicules). Les relations entre l'espace local-sud (secteurs de Bourg-en-Bresse et du Revermont) et l'espace régional-nord sont très faibles (85 véhicules) et orientées exclusivement vers Besançon. Pour ces quelques cas, l'autoroute A 39 pourra être utilisée entre les échangeurs de Viriat et de Bersaillin, mais au-delà, la RN 83 devrait garder un rôle prépondérant. Les flux entre Rhône-Alpes et l'espace local-nord sont un peu plus soutenus : $70 \%$ des 270 déplacements se font en relation avec l'agglomération lédonienne, $30 \%$ avec le secteur des plateaux jurassiens (Champagnole, Pontarlier), mais très peu avec le secteur dolois, dont la desserte vers le sud est déjà assurée par le réseau autoroutier existant (A 36 et A 6). Pour ces flux, la logique voudrait que l'A 39 soit bien empruntée entre la région lyonnaise et l'échangeur de Beaurepaire.

\section{Entre espaces locaux et espaces lointains}

16 Encore moins nombreux que les précédents, ces flux témoignent de la faiblesse des relations entre le milieu local et les autres régions françaises ou les pays étrangers. Au poste de Lons-nord, les relations entre l'espace local-nord et les espaces lointains-sud se font exclusivement avec les régions de la France méridionale (les pays étrangers, Italie et Espagne, ne sont pas cités). Elles sont d'ailleurs très peu nombreuses, moins de cent véhicules appartenant au secteur Arbois-Poligny, mais pourront utiliser l'A 39 à partir de l'échangeur de Bersaillin. Le secteur de Dole est logiquement absent, les relations vers le sud s'effectuant déjà par le réseau autoroutier existant (A 36 et A 6).

17 Pour les liaisons entre l'espace local-sud et les espaces lointains-nord, le trafic est plus important (237 véhicules). Les relations s'établissent entre la France du nord-est (mais sans pays étrangers) et dans l'ordre décroissant, les secteurs de Lons-le-Saunier, de Bourg-en-Bresse, puis des petites villes du Revermont. L'essentiel de ces flux devrait à l'avenir emprunter l'A 39, puis l'A 31 ou l'A 36.

\section{Les autres flux}

18 Ils doivent être considérés comme du transit pour lesquels les postes d'enquête rendent compte, l'un du trafic «montant», l'autre du trafic "descendant». En théorie, leurs valeurs sont identiques puisque les véhicules effectuent généralement l'aller et le retour d'un déplacement par le même trajet, les écarts à cette logique se compensant les uns les autres. Les chiffres globaux confirment cette hypothèse: le trafic "descendant» est de 557 véhicules au poste de Lons-nord tandis que le trafic 
«montant » est de 547 véhicules au poste de Lons-sud. À un niveau plus fin d'analyse, un tel équilibre n'est pas toujours respecté : aussi pour chaque type de flux et pour chaque poste, nous indiquerons les valeurs obtenues dans chaque sens, mais en termes de résultats nous retiendrons la valeur moyenne.

Les flux entre espaces régionaux nord et sud sont peu importants : 81 véhicules à Lonsnord, 102 à Lons-sud, soit, en moyenne, une petite centaine de déplacements. Au nord, ils concernent exclusivement la région bisontine. Au sud, la diversité est plus grande, même si l'agglomération lyonnaise est majoritairement présente. La faiblesse des échanges inter-régionaux par la RN 83 ne surprend pas dans la mesure où le réseau autoroutier existant assure déjà de bonnes relations entre ces deux espaces.

Les relations entre espaces régionaux et espaces lointains se divisent en deux flux. Le premier unit la région bisontine et les espaces lointains-sud représentés presque exclusivement par les régions du sud-est de la France; il est très faible : 89 véhicules à Lons-nord, 29 à Lons-sud (soit un chiffre moyen de 60 véhicules). Le second flux est plus abondant : 172 véhicules à Lons-nord, 210 à Lons-sud (moyenne 190). Il exprime les relations entre Rhône-Alpes (l'agglomération lyonnaise et la Savoie étant représentées à part égale) et la France du nord-est, avec une présence occasionnelle des pays étrangers.

21 Le dernier type de flux unit entre eux les espaces lointains nord et sud : les trafics sont de 215 véhicules pour Lons-nord et de 204 pour Lons-sud (moyenne 210). Vers le Nord les origines/destinations se répartissent entre la France (2/3) et l'étranger $(1 / 3)$. Vers le sud la France du sud-est domine, avec plus de $85 \%$ des flux, contre à peine $10 \%$ pour l'Espagne.

Pour l'ensemble des flux de transit, et quelles que soient leurs origines et destinations, le réseau autoroutier existant assure déjà une bonne desserte entre les différents espaces. L'enquête réalisée à la barrière de péage de Villefranche-sur-Saône et conduite dans des conditions similaires à celle de Lons-le-Saunier, donne pour les mêmes flux un total de 1500 véhicules. Le trafic relevé sur la RN 83 est donc loin d'être négligeable et son existence n'est pas due à un tracé inadapté du réseau autoroutier, mais plutôt à une volonté affichée de la part des utilisateurs de ne pas emprunter ce dernier. Les raisons de ce refus sont sans doute diverses : prix du péage, encombrement de l'A 6... Dans de telles conditions, il n'est pas sûr que l'ouverture de l'A 39 modifie profondément le comportement de ces usagers.

\section{Le flux de véhicules légers sur la RN 78}

Compte tenu de sa localisation, le poste de Lons-ouest présente beaucoup moins d'intérêt que les deux précédents, au regard d'éventuels apports de trafic à l'autoroute A 39. En effet la RN 78, sur lequel il est installé, est un axe essentiellement est-ouest, donc perpendiculaire au tracé général de l'A 39. Son trafic est de 2762 véhicules légers pendant la durée de l'enquête. L'organisation géographique des flux est marquée par un poids écrasant ( $96 \%$ du trafic total) des origines / destinations locales, qui ne concerneront pas l'A 39. Cet axe sert avant tout à deux types de trafics locaux : d'une part entre Lons-le-Saunier et sa zone d'influence périurbaine et rurbaine, d'autre part entre les petites villes bressanes à l'ouest (surtout Louhans) et la capitale jurassienne, 
mais aussi, secondairement, les petites cités industrielles du massif (Saint-Claude, Morez, Moirans-en-Montagne...).

Les autres types de flux sont faibles (125 véhicules toutes origines/destinations confondues) et là encore l'essentiel participe d'une logique est-ouest, entre les régions de la France du centre et de l'ouest et les espaces locaux jurassiens ou la Suisse par le col de la Faucille. Une trentaine de véhicules seulement pourraient emprunter la partie de l'A 39 située au sud de Lons-le-Saunier car ils correspondent à un flux entre la Bourgogne d'une part, le sud de l'Ain et la Savoie d'autre part.

\section{Les flux de véhicules poids-lourds}

Le trafic poids-lourds est surtout important le long de la RN 83 ; il est beaucoup plus faible sur la RN 78. La comparaison des résultats avec ceux obtenus pour les véhicules légers appelle trois remarques. Tout d'abord, et d'une façon générale, l'organisation géographique des flux est plus variée pour les poids lourds, avec une part importante des trafics de longue distance, alors que les relations strictement locales sont relativement modestes, ce qui met bien en évidence l'importance de la fonction de transit assurée par la RN 83 pour le transport des marchandises. Ensuite, le trafic international est important, surtout pour les échanges entre états du nord et états du sud de l'Europe. Enfin et toujours pour le trafic international, alors que pour les véhicules légers les relations vers le sud se faisaient presque exclusivement avec l'Espagne, pour les poids-lourds, l'Italie apparaît comme le partenaire majoritaire. Sans doute faut-il voir, dans cette situation originale, la conséquence de l'attitude de la Suisse qui refuse ce type de transit sur son territoire.

\section{Un bilan des transferts possibles}

Le redressement des résultats de l'enquête pour une journée moyenne de 24 heures et dans les deux sens, donne les fourchettes suivantes: entre 2000 et 3000 pour les véhicules légers, entre 800 et 1200 pour les poids-lourds, soit un total compris entre 2 800 et 4200 tous véhicules confondus.

Pour l'autoroute A 39, ces transferts issus du réseau routier classique ne devraient représenter qu'une partie minoritaire du trafic: l'essentiel sera fourni par les délestages provenant du réseau autoroutier existant (A 6, A 31, A 36). En outre, à terme, la circulation sur l'A 39 devrait être renforcée par les éventuels effets induits que sa présence peut provoquer sur le milieu local : apparition de nouvelles activités le long du tracé, acquisition de nouvelles habitudes de déplacement dans la population... Deux autres séries d'enquêtes, prévues en 1999 puis en 2003, devraient permettre d'apprécier la réalité des changements de comportements intervenus après une, puis quatre années de mise en service de la nouvelle infrastructure.

Enfin, la nouvelle répartition des flux de circulation entre les différents éléments du réseau devrait avoir d'importantes conséquences sur les axes délestés, en particulier sur la RN 83. D'une part, avec la diminution du trafic des véhicules légers, mais surtout des poids-lourds, les conditions de circulation devraient s'améliorer sensiblement en rapidité et en sécurité, au bénéfice des déplacements locaux. D'autre part, la disparition des flux de transit devrait perturber plus ou moins gravement le fonctionnement de 
certaines activités commerciales et de services (restaurants, garages, station-service...), qui en tiraient une partie de leur chiffre d'affaires.

\section{BIBLIOGRAPHIE}

Voir l'article de P. Bérion, en ce volume.

\section{NOTES}

1. Précisons que ce type d'enquête requiert au préalable une autorisation préfectorale et se déroule avec l'appui de la gendarmerie nationale qui dirige les véhicules sur les postes d'enquête.

\section{RÉSUMÉS}

L'ouverture, en juin 1998, de l'autoroute A 39 entre Dole (39) et Bourg-en Bresse (01) devrait modifier l'organisation des flux de circulation dans la région concernée par le nouvel axe de transport. L'exploitation des résultats d'une enquête auprès des usagers permet d'apprécier la nature et l'importance des modifications attendues.

The opening, in June 1998, of the A 39 motorway between Dole (39) and Bourg-en-Bresse (01) is planned to improve traffic flows, in the area of this new traffic axis. Results of a survey of road users facilitates assessment of the nature and importance of these long-awaited changes.

Die Eröffnung der Autobahn A39 zwischen Dole (39) und Bourg-en-Bresse (01) musste die Organisation der Verkehrsströme in dieser Region durch die neue Transportachse verändern. Die Auswertung einer Umfrage bei den Benutzern erlaubt es, Art und Umfang der zu erwartenden Veränderungen abzuschätzen

\section{INDEX}

Mots-clés : aménagement du territoire, autoroutes, flux Schlüsselwörter : Autobahnen, Régionalplannung, Ströme Keywords : flow, motorways, regional planning 


\section{AUTEUR}

\section{DANIEL MATHIEU}

IRADES@THEMA UPRESA 6049 du CNRS - Université de Franche-Comté - 32, rue Mégevand 25030 Besançon Cedex / Observatoire des effets économiques de l'autoroute A 39, SAPRR-SETRA, avec la collaboration du CETE de Lyon 